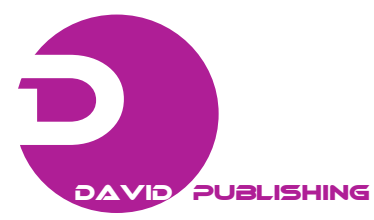

\title{
Direction Dependence of Savings on Cooling and Heating Loads by Energy Efficient Windows
}

\author{
Kazuki Yoshimura \\ Materials Research Institute for Sustainable Development, National Institute of AIST (Advanced Industrial Science and Technology), \\ Nagoya 463-8560, Japan
}

Received: August 30, 2014 / Accepted: October 10, 2014 / Published: December 31, 2014.

\begin{abstract}
The energy saving performance of energy efficient windows has strong dependence on window direction. Transmitted insolation level definitely affected the cooling and heating load. Simple simulation on the decrement of cooling load and the increment of heating load of a shading window compared with those of a transparent window show the prospect of energy saving effect clearly. From southeastward to southwestward, shading window even enlarges total heating and cooling loads when the thermal transmission is the same. However, if the shading coefficient of window is switched between summer and winter, total cooling and heating load can be reduced. This result clarifies the importance of "smart window".
\end{abstract}

Key words: Cooling load, heating load, shading coefficient, $U$-value.

\section{Introduction}

To reduce $\mathrm{CO}_{2}$ emission, many efforts have been taken in various fields. Window possesses the important position to reduce heating and cooling loads in buildings and vehicles. Some energy efficient windows, such as low-e glass have been commercialized and used. New smart windows which can be switched their optical and thermal properties have been expected to improve energy performance drastically.

Our group is now developing smart windows, such as switchable mirror window and thermochromic window. Not only material investigation, we are doing research to evaluate energy efficiency of smart windows [1].

Through these investigations, we notice that their energy performances have strong dependence on window direction, because sunshine condition is completely different from that of each direction. However, this direction dependence is not so well

Corresponding author: Kazuki Yoshimura, group leader, research field: preparation and characterization of functional thin film. E-mail: k.yoshimura@aist.go.jp. recognized among material scientists yet. In this paper, the author evaluates the direction dependence of energy performance of energy efficient windows.

\section{Methods}

Firstly, transmitted solar insolation through a window with specific shading coefficient is calculated as follows [2].

Solar elevation angle $h$ and solar azimuthal angle $A$ are given by:

$$
\begin{gathered}
\sin h=\sin \varphi \sin \delta+\cos \varphi \cos \delta \cos \omega \\
\cos A=\frac{\sin h \sin \varphi+\cos \varphi \cos \delta \cos \omega}{\cos h \cos \varphi}
\end{gathered}
$$

where, $\varphi=$ latitude; $\delta=$ declination of the sun; $\omega=$ hour angle.

$\delta$ is changed between $-23^{\circ} 27^{\prime}$ (the winter solstice) and $23^{\circ} 27^{\prime}$ (the summer solstice).

In this work, we are using the following approximation formula:

$$
\begin{gathered}
\sin \delta=\sin 23^{\circ} 27^{\prime} \cdot \sin B=0.397949 \sin B \\
B=360 \frac{n-81}{365}
\end{gathered}
$$

where, $B=$ circular orbit of the earth; $n=$ day of year. 
Hour angle $\omega$ is given by:

$$
\omega=\left\{t+\left(E+\frac{L-L S}{15}-12\right)\right\} \times 15
$$

where, $t=$ standard time; $E=$ equation of time; $L=$ longitude.

The following approximation formula is used for $E$ :

$$
E=\frac{1}{60}(9.87-7.53 \cos B-1.5 \sin B)
$$

In this paper, all calculations are for Nagoya (Japan); the longitude is $139.77^{\circ}$ and the latitude is $35.65^{\circ}$, respectively.

After the determination of the sun position; $h$ and $A$, insolation level is estimated as follows.

Extraterrestrial solar radiation $I_{0}$ is given:

$$
I_{0}=I_{S C}\left\{1+0.033 \cos \left(\frac{2 \pi n}{365}\right)\right\}
$$

where, $I_{S C}=$ solar constant $=1,370 \mathrm{~W} / \mathrm{m}^{2}$.

Normal direct insolation $I_{D N}$, diffuse sky radiation on a horizontal surface $I_{S K Y}$, and global solar radiation on a horizontal surface $I_{H O L}$ are important parameters for insolation. They are given by:

$I_{D N}$ is determined by:

$$
I_{H O L}=I_{D N} \sin h+I_{S K Y}
$$

$$
I_{D N}=I_{0} P^{1 / \sin h}
$$

where, $P=$ atmospheric transmissivity. In this paper, the empirical value of $P$ in Nagoya (0.62-0.8) is used for calculation.

$I_{S K Y}$ is given as follows by using Nagata's equation:

$$
\begin{gathered}
I_{S K Y}=\sin h\left(I_{0}-I_{D N}\right)(0.66-0.32 \sin h) \\
\{0.5(0.4-0.3 P) \sin h\}
\end{gathered}
$$

Separating of direct and diffuse insolation is done using the method proposed by Udagawa and Kimura [2] as follows.

$I_{D N}$ is recalculated by the following formula:

$$
\begin{gathered}
I_{D N}=\left(-0.43+1.43 K_{T t}\right) I_{0} \\
\left(K_{T t} \geq K_{T t C}\right) \\
I_{D N}=\left(\begin{array}{c}
2.277-1.258 \sin h \\
+0.2396 \sin ^{2} h
\end{array}\right) K_{T t}^{3} I_{0}\left(K_{T t}<K_{T t C}\right)
\end{gathered}
$$

where,

$$
K_{T t}=\frac{I_{H O L}}{I_{0} \sin h}
$$

$$
K_{T t C}=2.277-1.258 \sin h+0.2396 \sin ^{2} h
$$

Then, $I_{S K Y}$ is recalculated using the following formula:

$$
I_{S K Y}=I_{H O L}-I_{D N} \sin h
$$

Insolation to window $I_{W}\left(\mathrm{~W} / \mathrm{m}^{2}\right)$ is the summation of direct insolation $I_{D}$ and diffuse insolation $I_{d}$ :

$$
I_{W}=I_{D}+I_{d}=I_{D}+\left(I_{S}+I_{R}\right)
$$

where, $I_{S}=$ diffuse sky radiation; $I_{R}=$ reflected solar radiation.

Direct insolation $I_{D}$ is given by:

$$
I_{D}=I_{D N} \cos \theta
$$

where, $\theta=$ incident angle of direct sunshine to window.

$I_{S}$ and $I_{R}$ are calculated by the following formula:

$$
\begin{gathered}
I_{S}=0.5 I_{S K Y} \\
I_{R}=0.5 \rho_{G} I_{H O L}
\end{gathered}
$$

where, $\rho_{G}$ is albedo, which is supposed to be 0.5 in this paper.

After the determination of insolation to window, transmitted insolation $I_{T}$ through the window with a specific SC (shading coefficient) is calculated by:

$$
I_{T}=\left(I_{D} \times\left(1-F_{R}\right)+I_{d}\right) \times S C
$$

where, $F_{R}$ is Fresnel reflection index.

Generally, the relationship between transmitted insolation and heating and cooling loads is very complicated. Because the purpose of this paper is to obtain a design guide of energy performance of smart widows, this relationship is boldly simplified as follows.

Cooling energy is mostly consumed in August and heating energy is mostly consumed in February. Hence, the total heating load is subsumed to be rational to the averaged difference of transmitted insolation between shading window and transparent window in February. Similarly, the total cooling load is assumed to be rational to the averaged difference between shading window and transparent window in August. By calculating these values with respect to shading coefficient, the total heating and cooling load through a year is evaluated by the balance of the decrement of cooling load and the increment of heating load. Also, the direction dependence of the balance is evaluated. 


\section{Results and Discussion}

Fig. 1 shows the calculated variation of vertical insolation and transmitted insolation through 3-mm thick transparent glass (reference of shading coefficient) to the south-faced window. This graph shows that insolation in winter is much larger than that in summer for the just south direction. This difference is enlarged by Fresnel reflection for the transmitted insolation.

Fig. 2 shows day variations of the transmitted insolation on January 1st and July 1st, which are picked up from Fig. 1. It shows that although daylight hours are longer in summer, radiation level is about three times larger in winter than that in summer. The integrated insolation of the day is $4,376 \mathrm{Wh} / \mathrm{m}^{2}$ on January $1 \mathrm{st}$ and $1,490 \mathrm{Wh} / \mathrm{m}^{2}$ on July $1 \mathrm{st}$, respectively.

On the other hand, Fig. 3 shows the variation of vertical insolation and the transmitted insolation through 3-mm thick transparent glass to the west-faced window. To west direction, the insolation in summer is larger than that in winter.

Fig. 4 shows the day variations of the transmitted insolation on January 1 st and July $1 \mathrm{st}$, which are picked up from Fig. 3. The integrated insolation of the day is $1,403 \mathrm{Wh} / \mathrm{m}^{2}$ on January $1 \mathrm{st}$ and $3,566 \mathrm{Wh} / \mathrm{m}^{2}$ on July 1st, respectively. The insolation in summer is about 2.5 times larger than that in winter.

From these values, transmitted insolation through 3-mm thick transparent glass (reference) and shaded glass with a specific $S C$ are calculated. The shading reduces the cooling load in summer, while the shading enlarges the heating load in winter. Total energy saving through a whole year of a shading window is determined by the balance of the decrement of cooling load and the increment of heating load compared with a transparent window.

Fig. 5 shows the effect of shading for south-faced window. The solid line is the averaged difference of transmitted insolation per square meter per day between shading window with specific shading coefficient value and transparent window with 3-mm thick glass in August, which indicates the decrement of

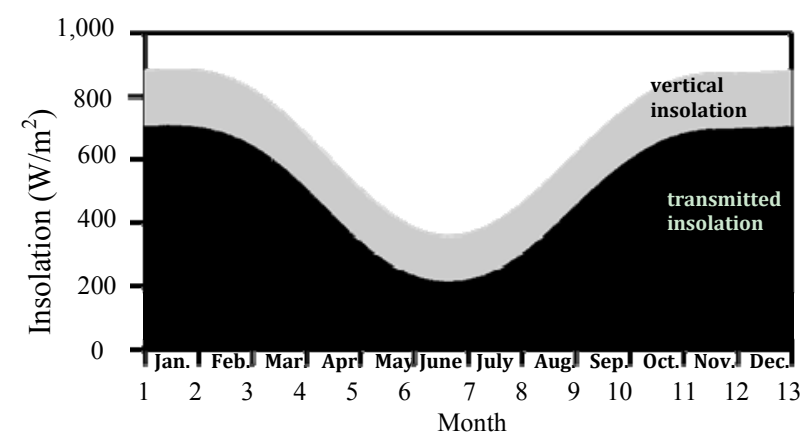

Fig. 1 Vertical insolation and transmitted insolation through 3-mm thick transparent glass to south-faced window.

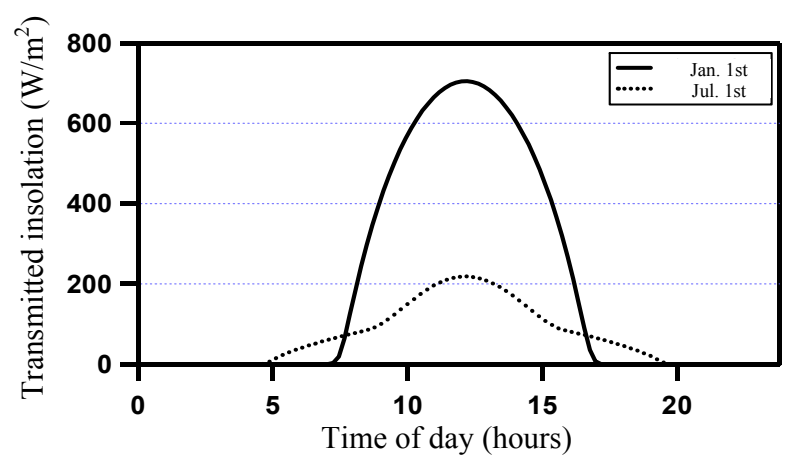

Fig. 2 Day variation of the transmitted insolation on January 1st and July 1st to south-faced window.

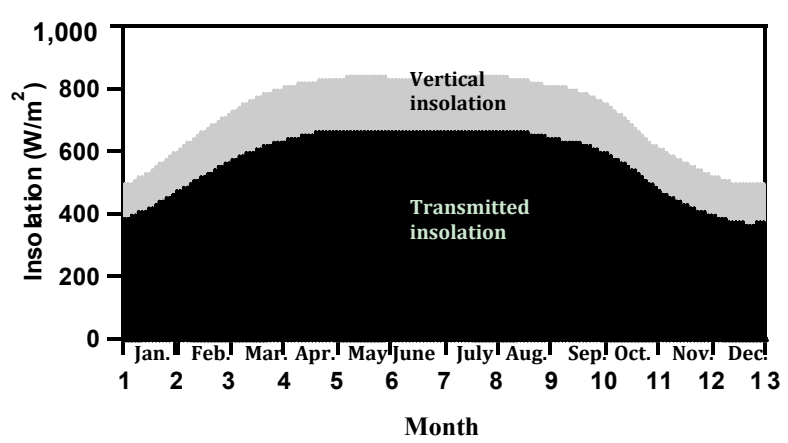

Fig. 3 Vertical insolation and transmitted insolation through 3-mm thick transparent glass to west-faced window.

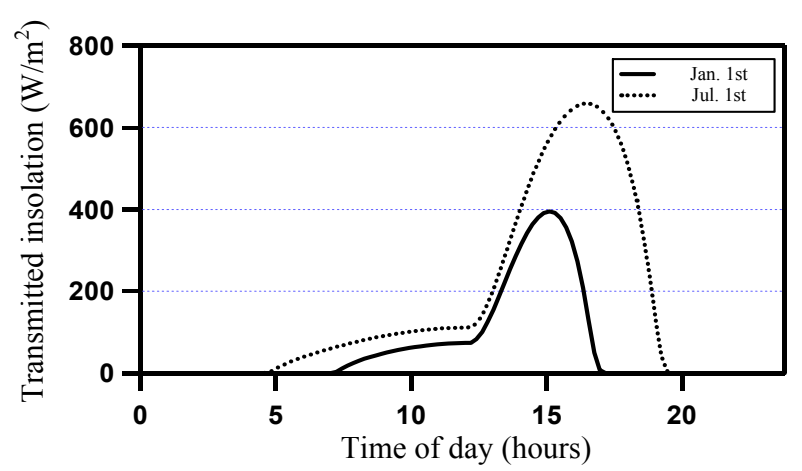

Fig. 4 Day variation of the transmitted insolation on January 1st and July 1st to west-faced window. 


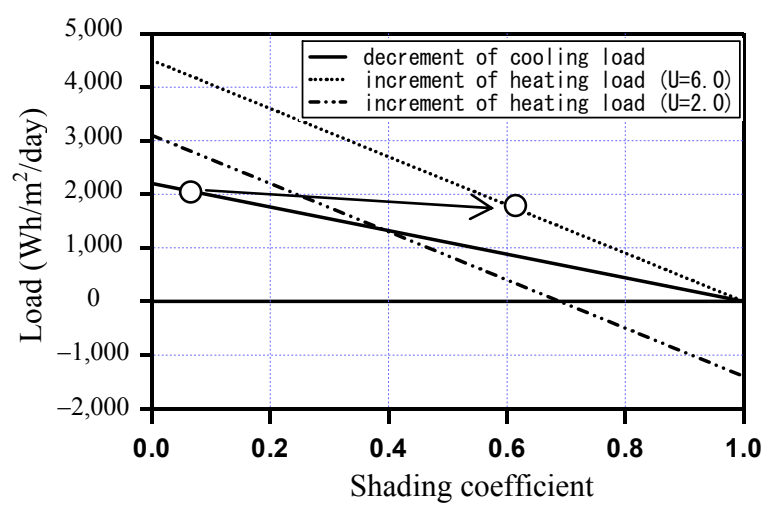

Fig. 5 Effect of shading for south-faced window.

cooling load in summer. The dotted line is the averaged difference of transmitted insolation between shading window and transparent window in February, which indicates the increment of heating load in winter.

This graph shows that the decrement of cooling load is increasing with decreasing shading coefficient. However, the increment of heating load is increasing much larger than the decrement of cooling load, because the insolation in winter is much larger than that in summer, as shown in Fig. 2. So, the total heating and cooling load through a whole year is increasing instead of decreasing. For example, in the case of the shading window with $S C=0.55$, the increment of cooling load is $990 \mathrm{Wh} / \mathrm{m}^{2} /$ day, while the decrement of heating is $1,950 \mathrm{Wh} / \mathrm{m}^{2} /$ day. The total heating and cooling load is enlarged of $960 \mathrm{Wh} / \mathrm{m}^{2} /$ day. This result shows that the energy performance is the best for the completely transparent window, when the thermal transmission is the same.

The energy performance of window is determined by shading coefficient as well as $U$ ( $U$-value) which indicates thermal transmission. Taking into account thermal transmission, these curves should be modified. In Nagoya, the average temperature is $5{ }^{\circ} \mathrm{C}$ in February and $28{ }^{\circ} \mathrm{C}$ in August, respectively. I assume that the room temperature is kept at $20{ }^{\circ} \mathrm{C}$ in winter and $28{ }^{\circ} \mathrm{C}$ in summer by air conditioning, respectively. The $U$-value of 3-mm thick glass is 6.0. Using the window with low $U$-value, the heating load is reduced by:

$$
(20-5) \times(6-U) \times 24\left(\mathrm{Wh} / \mathrm{m}^{2} / \text { day }\right)
$$

The average temperature and air conditioning temperature is almost the same in August and the thermal flow is negligible.

The increment of heating load for the window with $U=2$ is plotted in Fig. 5 by the dashed line. In this condition, for example, the decrement of cooling load is $990 \mathrm{Wh} / \mathrm{m}^{2} /$ day for the window with $S C=0.55$, which is applicable for a practical low-e glass. The increment of heating load is reduced to $650 \mathrm{Wh} / \mathrm{m}^{2} /$ day, and the total heating and cooling load can be reduced, although the saving level is not so large.

Fig. 5 also shows that the total cooling and heating load can be reduced if the shading coefficient of glass can be switched between winter and summer even when thermal transmission is the same. For example, if the case of the window with $S C=0.1$ in winter and with the $S C>0.55$ in summer, the total cooling and heating load is reduced compared with transparent window. In our group, we are developing new smart window- -switchable mirror window". This window can be switched between $S C=0.06$ in summer and $S C$ $=0.63$ in winter [3], which are plotted in Fig. 5 . In this case, the decrement of cooling load is larger than the increment of heating load and the total cooling and heating loads can be reduced.

On the other hand, effect of shading for west-faced window is direct opposition to that of south-faced window, as shown in Fig. 6. With decreasing of shading coefficient, the decrement of cooling load is increasing. This value is larger than that of the increment of heating load. It means that the shading coefficient is getting smaller, the energy saving is getting larger. Shading is effective to reduce the total heating and cooling load in this direction. But it should be noted that no window is the best energy performance.

If the shading coefficient of window can be switched widely between summer and winter, energy efficiency can be improved. For example, if the case of the window with $S C=0.06$ in winter, and with $S C=0.63$ in summer by using switchable mirror window, which 


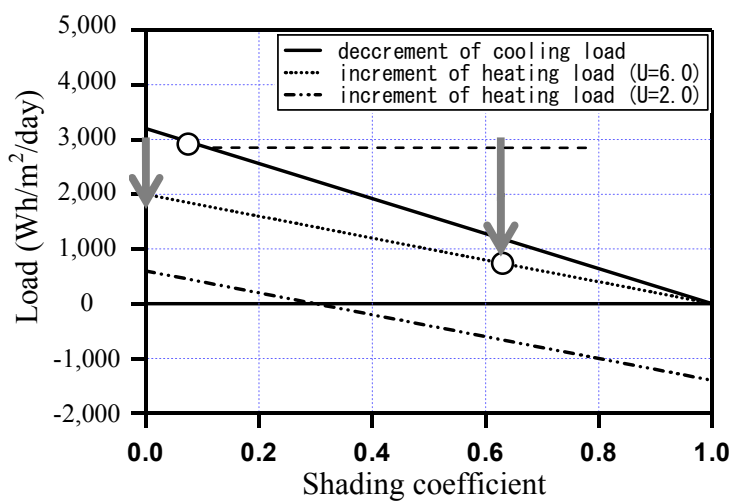

Fig. 6 Effect of shading for west-faced window.

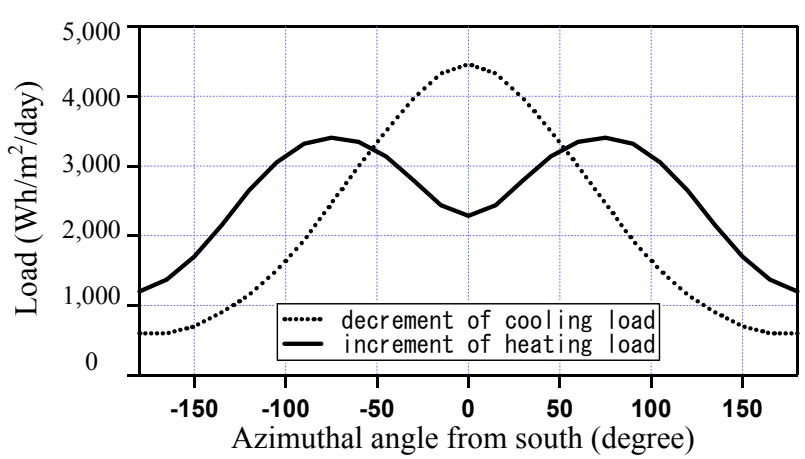

Fig. 7 Decrease of cooling load and the increase of heating load at fully shaded state with respect to the azimuthal angle from south.

are plotted in Fig. 6, the total cooling and heating load is reduced compared with the case of no window.

Other direction is the intermediate state between south and west directions. The decrement of cooling load is increasing with deviation from directly south. The values of the increment of the heating load and the decrement of the cooling load at fully shaded state ( $S C$ $=0$ ) are good indicators to see the effect of shading. Fig. 7 shows the dependence of shading effect for other direction. Eastward direction and westward direction are almost symmetrical

From southwest to southeast, the increment of heating load is larger than the decrement of cooling load. In these directions, transparent window has better energy performance. At about $50^{\circ}$ from south, the decrement of cooling load and the increment of heating load are balanced. In other directions, the decrement of cooling load is larger than the increment of heating load and shading is effective to reduce the total heating and cooling load.

\section{Conclusion}

Effect of shading of window on the total heating and cooling load is investigated by the simple simulation.

This evaluation reveals that the required shading performance of energy efficient window has strong dependence on window direction from the view point of the saving of heating and cooling load through the year.

From southeastward to southwestward, shading window even enlarges total heating and cooling loads, when the thermal transmission is the same. However, if the shading coefficient of window is switched between summer and winter, total cooling and heating load can be reduced.

Although the shading window can reduce the total cooling and heating load for other directions, its energy saving is smaller than that of the case of no window. If the shading coefficient is changed between summer and winter widely, the total cooling and heating loads can be reduced. These results clarify the importance of "smart window".

\section{References}

[1] Yoshimura, K., Yamada, Y., Bao, S., Tajima, K., and Okada, M. 2009. "Preparation and Characterization of Gasochromic Switchable-Mirror Window with Practical Size.” Solar Energy Materials and Solar Cells 93: 2138-41.

[2] Udagawa, M. 1986. Simulation Method of Air Conditioning by Personal Computer. Ohm-sha.

[3] Yoshimura, K., Langhammer, C., and Dam, B. 2013. "Metal Hydrides for Smart Window and Sensor Applications." MRS Bulletin 38: 495-503. 